\title{
EL PAPEL DE LAS VEEDURÍAS CIUDADANAS EN COLOMBIA
}

\section{THE ROLE OF CITIZEN OVERSIGHT IN COLOMBIA}

\author{
Julián Cocuy Espinosa, Piedad Fernanda Machado Santacruz y Daniela Montoya Ortiz \\ Semillero Gestión E \\ Grupo de investigación GICES \\ Institución Universitaria Antonio José Camacho \\ Cómo citar este artículo: \\ Cocuy Espinosa, J., Machado Santacruz, P.F., Montoya Ortiz, D. (2021). El papel de las veedurías ciudadanas en Colombia. Revista \\ Sapientía, 13(26), 43-50.
}

\section{RESUMEN}

Reconociendo la importancia de los derechos y los mecanismos de participación ciudadana, este artículo da a conocer la veeduría en Colombia, la cual es uno de los tantos mecanismos de participación para que el ciudadano pueda vigilar e informarse sobre las distintas entidades privadas y públicas que manejan recursos del Estado. El contexto histórico alrededor de este mecanismo es importante para entender el desarrollo de la democracia en Colombia y la importancia del rol de la ciudadanía en el desarrollo y progreso del Estado. Gracias a la reforma que trajo consigo la Constitución Política de Colombia, la veeduría se considera como uno de sus principios fundamentales en la democracia participativa.

\section{PALABRAS CLAVE}

Democracia, participación ciudadana, veeduría ciudadana.

\section{ABSTRACT}

Recognizing the importance of the rights and the mechanisms of citizen participation, this article presents the oversight in Colombia, which is one of the many participation mechanisms so that the citizen can monitor and learn about the different private and public entities that manage State resources. The historical context around this mechanism is important to understand the development of democracy in Colombia and the importance of the role of citizens in the development and progress of the State. Thanks to the reform that the Political Constitution of Colombia brought with it, oversight is considered one of its fundamental principles in participatory democracy.

\section{KEYWORDS}

Democracy, Citizen Participation, Citizen Oversight.

\section{INTRODUCCIÓN}

El proyecto de investigación: Sistematización de experiencias de convenio marco de cooperación entre la Contraloría Municipal de Yumbo y la Institución Universitaria Antonio José Camacho, para la capacitación y asesoría en el fortalecimiento del ejercicio veedor de la comunidad del municipio (2020-PI-0521), avalado y patrocinado por la Institución Universitaria Antonio José Camacho y la Contraloría Municipal de Yumbo, el cual fue aprobado mediante Resolución de la UNIAJC No. 116 de febrero 8 de 2021: Por medio de la cual se da 
aval y se asigna presupuesto a unos proyectos de investigación de la UNIAJC, aborda temas como la veeduría ciudadana mediante la cual la población, como uno de los elementos principales que conforman el Estado, tiene un papel crucial en todos los ámbitos y le compete directamente todas las decisiones tomadas por esta organización; por lo tanto, que la población se involucre activamente en el proceso de la toma de decisiones es completamente natural e inevitable (Arboleda, Cuellar, \& Chantre, 2021).

Según el artículo primero de la Constitución Política de Colombia de 1991, el Estado se declara como una república democrática, participativa y pluralista, constituyendo como derecho fundamental el hecho de que todos los elementos del Estado son partes activas del mismo y que pueden incursionar de manera organizada en las cuestiones que lo aquejen, lo cual es necesario para que la democracia sea posible. Este proceso se denomina participación ciudadana.

En el presente texto se buscará exponer uno de los mecanismos que ha establecido la ley para hacer efectivo el cumplimiento de este derecho fundamental, el cual se puede explicar como la modalidad de participación de la ciudadanía dentro de la gestión pública, en donde su desarrollo debe ser de dominio público, mostrando sus resultados. Todo esto tiene como punto de partida el marco legal existente, junto con el marco histórico de la nación en cuanto al desarrollo de la participación desde la época de la Constitución de 1886, pasando por la Constitución de 1991 hasta hoy.

\section{MARCO TEÓRICO Antecedentes}

La veeduría ciudadana en Colombia toma importancia a partir de la promulgación de la Constitución Política de Colombia de 1991, su función principal es contribuir en la vigilancia y control del manejo del recurso público de aquellas entidades públicas o privadas que manejan y ejecutan el erario, para mejorar la administración de la gerencia pública.

La Constitución Política de la República de Colombia de 1991 hace un reconocimiento a los procesos democráticos y a la participación ciudadana, dándole al ciudadano herramientas para que puedan ejercer sus derechos y obligaciones, y así intervenir de manera activa en la gestión pública.

En el marco de los antecedentes de esta temática, en el trabajo de grado "Veeduría al Decreto 120 del 2007 y el proceso de desarrollo urbano del municipio de Fusagasugá (Cundinamarca)", Suárez (2017) se propone identificar la importancia del estudio del Decreto 120 de 2007 con relación al proceso urbano del municipio de Fusagasugá, este decreto tuvo implicaciones con relación al desarrollo paulatino del modelo de ciudad. El enfoque empleado fue mixto, al contener metodología cualitativa y cuantitativa beneficiando la investigación hacia la aproximación del panorama social de los ciudadanos. La recolección de los datos se realizó con base en fuentes documentales, uso de software y entrevista semiestructurada que fue dirigida hacia el ciudadano que constituyó la veeduría ciudadana del Decreto 120 de 2007. El análisis de los resultados permitió evidenciar que se presentaron irregularidades en el Plan Ordenamiento Territorial, el cual afectaba directamente la participación ciudadana de la población del Municipio de Fusagasugá, quienes no podrían acceder al suelo municipal (Suárez, 2017).

\section{MARCO HISTÓRICO DE LA PARTICIPACIÓN CIUDADA- NA ANTES DE LA CONSTITUACIÓN POLÍTICA DE 1991}

Uno de los puntos más importantes en el contexto del Estado es el desarrollo de la democracia a través de la historia. La vieja disputa partidista entre conservadores y liberales provocaron una crisis que afectó la legitimidad de la democracia, pues llevaron a la nación a un escenario en el cual la participación era de manera simbólica, pasiva, es decir, alejada de los ciudadanos, quienes tenían solamente el voto como medio para manifestar su opinión. 
Sin embargo, gracias a esa disputa, una gran cantidad de cambios constitucionales y sociales desencadenaron la constitución de 1991. La Constitución Política de 1886 era menos estricta, lo que permitía que el gobierno figurara como una entidad que podía actuar bajo sus propios intereses.

El gobierno no tenía la presión de la población que demandara estar a su servicio. Tal como se dijo antes, el pueblo no poseía un medio por el cual se pudiese escuchar su voz en situaciones que le aquejaban siendo opacado por los intereses de las mayorías. Este hecho se acentuaba mucho más debido al conflicto entre los dos partidos y a la supresión de toda posible vertiente que atentara contra el orden impuesto, como lo fueron en su momento el Partido Comunista, las ideas progresistas de Jorge Eliécer Gaitán y la Alianza Nacional Popular (ANAPO) de Gustavo Rojas Pinilla, que constituyen los primeros indicios del deseo latente en el país por un cambio en el que la población se viera fuertemente representada y tuviera, por consiguiente, un papel importante.

Estas supresiones causaron en muchas ocasiones respuestas violentas por parte de la población debido al descontento frente a esta situación. Esta tendencia se mantuvo durante mucho tiempo. Finalizando la primera mitad del siglo $\mathrm{XX}$, en búsqueda de la pacificación institucional, los partidos tradicionales conformaron el denominado Frente Nacional (1.958-1974) el cual era un acuerdo político en el que se concertaba la alternancia del poder durante cuatro años con el propósito inicial de que cesara la violencia y un fortalecimiento del Estado, el Frente Nacional inicio con el mandato del presidente liberal Alberto Lleras Camargo. Este acuerdo generó una práctica limitada de la democracia, la cual pasó a ser vista como restringida y excluyente.

Gracias a esta concepción representativa de la democracia, se redujeron drásticamente las condiciones de participación conduciendo a un estancamiento en el modelo institucional, lo cual generó una pérdida de legitimidad en los ciudadanos frente al voto y a la idea de democracia representativa que no daba respuestas a los reclamos de los sectores minoritarios.

El descontento de la población comenzó a evidenciarse en la manifestación de insurgencias que promovían la necesidad de cambios políticos profundos. Esto permitió que la Asamblea Nacional Constituyente de 1990 hiciera una nueva construcción de la democracia a partir de términos participativos, permitiendo la concepción de formas diferentes de participar democráticamente a parte del proceso electoral.

De este modo se inicia la construcción de una nueva institucionalidad, en donde los constituyentes abrieran paso a nuevas opciones políticas donde el principal objetivo era incluir la dimensión participativa en la democracia colombiana.

Con la llegada de la Constitución Política de 1991 la democracia pasó de ser representativa solamente, a incluir de manera activa a la población en los asuntos del Estado, a través de la legislación de los nuevos mecanismos de participación consagrados en el artículo 103 de la carta política, lo que permitió una visión completamente distinta a la anterior de lo que era el Estado y se escuchaban al fin a los grupos de personas organizadas que buscaban defender sus derechos.

Con el cambio constitucional, aparecen en la política nuevos actores cívicos, sociales, étnicos y económicos que antes no eran considerados por los gobiernos debido a su condición minoritaria, para ser reconocidos en su valor individual evidenciando un desarrollo más amplio en términos de democracia y participación acorde a los principios fundamentales establecidos en el Título I de la Constitución Política de Colombia (Arboleda, Cuellar, \& Chantre, 2021).

Esta transformación es la concepción de participación gracias a los cambios incorporados en las normas 
jurídicas y estos se entienden por medio de una aplicación que va más allá del carácter legalista. La Constitución Política de Colombia fundamenta al Estado desde una base que tiene como cimiento la participación de todos los actores que influyen en su funcionamiento, incluyendo toda la población.

Por otro lado, queda entonces que la constitución reconoce un papel activo tanto del Estado como de la ciudadanía, siendo el Estado el ente regulador y la ciudadanía el actor que colabora alrededor de una construcción más efectiva de la democracia.

Entre la democracia participativa y representativa surge una relación de cooperación del Estado y la ciudadanía lo que permite definir dos formas de participación: primero la participación representativa o indirecta, la cual se evidencia en los procesos democráticos de elección popular de presidente, congresistas y demás cargos que sean sometidos a la decisión del constituyente primario, de elección popular y segundo la participación directa que se puede identificar como el medio por el cual las comunidades, minorías y todos los actores sociales pueden manifestarse. Mediante estos mecanismos de participación democrática se busca reconocer como la situación social, cultural, económica o política del Estado está relacionada con la población del país.

Aunque estos dos modelos trabajan, de manera conjunta la participación directa, tienen una posición más dirigida a la personalización de la democracia, encaminada a la toma de decisiones específicas que vinculan más estrechamente a la ciudadanía, ya que la participación no es más que la posibilidad de un individuo que hace parte de la comunidad a la cual pertenece.

\section{FUNDAMENTOS CONSTITUCIONALES Y LEGAL DE LAS VEEDURÍAS CIUDADANAS EN COLOMBIA}

Las veedurías ciudadanas son un mecanismo de participación ciudadana, las cuales se encuentran reguladas por la Constitución Política de Colombia de 1991 y sus desarrollos legislativos:
- Ley 134 de 1994. Mecanismos de participación ciudadana

- Ley 489 de 1998. Ejercicio de la Administración Pública

- Ley 850 de 2003. Veedurías Ciudadanas

- Ley 1474 de 2011. Estatuto Anticorrupción

- Ley 1757 de 2015. Estatuto de la Participación ciudadana

Este mecanismo de participación ciudadana contempla los siguientes objetivos en el Artículo 6 de la Ley 850 de 2003:

1. Erradicar la corrupción mediante los mecanismos de participación y el control de la gestión pública y contrataciones.

2. Enriquecer el poderío de la ciudadanía y generar en ellos una interacción directa en la toma de decisiones públicas y en el seguimiento e interferencia de los proyectos de inversión.

3. Fomentar los ejercicios de las personerías municipales con respecto a la promoción de la participación comunitaria.

4. Velar por el interés general de la ciudadanía cómo beneficiaros de la acción pública.

5. Disponer el cumplimiento de los principios constitucionales que se estipulan en la función pública.

6. Generar una participación directa de los particulares en la administración de los altos cargos con el fin de evitar el abuso de poder y el mal uso de los bienes públicos.

7. Democratizar la gestión pública. 


\section{Invitar al liderazgo y la internación comunitaria.}

Acorde a lo anterior, la vigilancia ejercida por las veedurías ciudadanas se realiza sobre la gestión administrativa, teniendo en cuenta el valor del interés general y principios delimitados por la ley.

\section{CONSTITUCIÓN, PROCEDIMIENTOS Y EJERCICIO DE LAS VEEDURÍAS CIUDADANAS}

Según el Artículo 2 de la Ley 850 de 2003, el cual comprende la facultad de constitución, todas las personas de forma plural o a través de organizaciones sin ánimo de lucro podrán ejercer la veeduría ciudadana.

Las acciones que competen al procedimiento para participar en los procesos de la veeduría ciudadana están comprendidas en el Artículo 3 de la Ley 850 de 2003 y consagra que las personas que desean participar en la veeduría ciudadana, conforme al artículo anterior, deberán elegir a un representante o a un veedor o veedores, estos deberán presentar un documento en el que se especifiquen los documentos de identidad, lugar de residencia, el objetivo de la vigilancia y el nivel territorial.

Este documento deberá ser presentado ante las personerías municipales o distritales o a la Cámara de Comercio con el fin de llevar registro público de las veedurías inscritas en su registro.

En caso de ser una veeduría solicitada por parte de la comunidad indígena o que desee realizarse en dicha comunidad será procedida y asumida por las autoridades propias de la comunidad.

Por otro lado, según el Artículo 5 de la Ley 850 de 2003, las veedurías podrán ser ejercidas en cualquier entidad que se ubicase sobre la nacionalidad territorial y la gestión pública, ya sean entidades centralizadas o descentralizadas respecto a la administración pública. Las entidades descentralizadas creadas de forma indirecta por empresas con participación en el capital público y privado también podrán ejercer la veeduría ciudadana.

\section{METODOLOGÍA}

La recopilación y el análisis del desarrollo de este artículo exigió la utilización del método deductivo siendo la dinámica más apropiada, porque permite partir de lo general a lo particular, tomando como referente el desarrollo conceptual, histórico constitucional y legal de los mecanismos de participación ciudadana y el ejercicio democrático de las veedurías en Colombia.

\section{MÉTODO DE INVESTIGACIÓN}

El método de la investigación describe con buenos detalles la forma en que se ha llevado a cabo la investigación. Este método permite explicar la propiedad de los métodos utilizados y la validez de los resultados, incluyendo la información pertinente para entender y demostrar la capacidad de replicación de los resultados de la investigación. El método cualitativo, en la aplicación para la investigación en las ciencias sociales continúa descubriendo conceptos que no son evidentes, al mismo tiempo presenta una oportunidad para presenciar nuevas perspectivas para aquellas situaciones en las que ciertas señales ocultas revelan un mayor conocimiento del fenómeno de investigación (Abreu, 2014).

\section{TIPO DE ESTUDIO}

El tipo de estudio apropiado para esta investigación fue descriptivo porque permitió definir y catalogar los temas principales de este documento. Díaz (2009) afirma: "Los estudios descriptivos buscan especificar propiedades importantes de personas, grupos, comunidades o cualquier otro fenómeno que sea sometido a análisis. Miden o evalúan diferentes aspectos, dimensiones o componentes del fenómeno a investigar" (p.180). Un estudio descriptivo tiene información sobre los diferentes temas y son sometidos a un análisis posterior, el cual permitirá la descripción de la investigación.

\section{ENFOQUE DE INVESTIGACIÓN}

Este documento tiene un enfoque cualitativo, el cual muestra referencias sobre el contexto histórico de 
participación ciudadana antes de la Constitución Política de 1991, el concepto de veeduría ciudadana, así como el marco constitucional, normativo y legal.

\section{FUENTES DE INVESTIGACIÓN}

La información se recolectó a través de diferentes sitios web, páginas oficiales de instituciones del Estado colombiano, documentos, artículos y demás referentes académicos.

\section{TÉCNICAS DE RECOLECCIÓN DE INFORMACIÓN}

La técnica de recolección empleada fue el análisis documental que permitió la recuperación y recopilación de la información.

\section{RESULTADOS}

Reconocer los derechos y obligaciones de los ciudadanos es fundamental para un Estado Social de Derecho. La Constitución Política de Colombia de 1991, que derogó la de 1886, reconoce y valora las diferentes formas de participación ciudadana, introdujo importantes cambios en materia constitucional con relación a las formas y sistemas de participación ciudadana, que tienen por objeto la vigilancia de la gestión pública, fiscal y financiera.

En los últimos diez años, ha sido indispensable el papel que han ejercido las veedurías ciudadanas, porque permiten que los habitantes, o las diferentes organizaciones de tipo (profesional, comunitaria, juvenil, entre otras) se constituyan sin ánimo de lucro y sean utilizadas como una herramienta que procure el bien común. Por lo tanto, desde la creación de estas, la población tendrá la facultad de presentar actas de conformación, que señalen el objeto a vigilar con respecto a la gestión pública, tanto de entidades públicas, como privadas (Machado \& Montoya, 2021).

El concepto de veeduría ciudadana en Colombia no había tenido un desarrollo institucional que permitiera definir de manera específica la participación ciudadana en el control de la gestión de los bienes y servicios. Sin embargo, tras la expedición de la Constituci- -ón Política de 1991, la participación ciudadana empezó a tener un papel más dinámico en la sociedad propiciando mayor interés en la población por temas referentes a sus derechos y obligaciones.

\section{CONCLUSIONES}

En Colombia, los procesos que hicieron parte del desarrollo de los mecanismos de participación ciudadana se encuentran consignados en la Constitución Política de Colombia de 1991, que les otorga a los ciudadanos las herramientas de rango constitucional para la vigilancia de la gestión pública.

Las veedurías ciudadanas se institucionalizaron para que los ciudadanos puedan vigilar, inspeccionar, controlar la administración y gestión pública, además, son de carácter cívico, voluntario, independiente y neutral.

Al ser organizaciones sin ánimo de lucro y con carácter altruista no persiguen intereses o beneficios personales, son de carácter temporal y no constituyen órganos de dependencia del Consejo de Participación Ciudadana y Control Social ni de la entidad observada, siendo su objetivo central la prevención de actos de corrupción.

La comunidad de vigilancia podrá realizar su ejercicio veedor a las diferentes instituciones del estado o particulares que administren fondos o recursos públicos de la nación, bajo los siguientes criterios:

- Asignación de recursos

- Aplicación de recursos

- Ejecución de los planes, programas y proyectos

- Cobertura y calidad de los servicios públicos

- Efectividad y oportunidad en la prestación de los servicios

Para el ejercicio de la veeduría ciudadana y afinando el procedimiento que se debe realizar, la Constitución Política de Colombia estableció mecanismos para facil- 
itar a la comunidad de vigilancia las siguientes herramientas:

- Derecho de petición

- Acciones judiciales

- Audiencias públicas

- Denuncias y quejas

- Instrumentos legales

- Control excepcional

El control excepcional es una alternativa que puede solicitar una veeduría a la Contraloría General de la República para realizar intervención o control en un proceso, obra, proyecto o contrato.

\section{REFERENCIAS BIBLIOGRÁFICAS}

Abreu, J. L. (2014). El método de la investigación. International Journal of Good Conscience, 9 (3), 195-204.

Arboleda, A. A., Cuellar, M. \& Chantre, A. K. (abril de 2021). Veeduría Ciudadana en Colombia. Universidad del Valle-Cátedra Constitución Política.

Constitución Política. (05 de agosto de 1886). Vista la aprobación que impartieron las Municipalidades de Colombia a las bases de Constitución expedidas el día $1 .^{\circ}$ de diciembre de 1885. https://www.funcionpublica.gov .co/eva/gestornormativo/norma.php?i=7153.

Constitución Política de la República de Colombia. (20, de julio de 1991). Esta versión corresponde a la segunda edición corregida de la Constitución Política de Colombia, publicada en la Gaceta Constitucional No. 116 de 20 de julio de 1991. http://www. secretariasenado.gov.co/senado/basedoc/const itucion_politica_1991.html.

Díaz, N. V. (2009). Metodología de la investigación, científica y bioestadística. Ril editores.

Ley 134. (31 de mayo de 1994). Por la cual se dictan normas sobre mecanismos de participación ciudadana. Diario Oficial 41.373. Bogotá, Colombia: Recuperado de: http://www .secretariasenado.gov.co/senado/basedoc/ley_ 0134_1994.html.

Ley 1474. (12 de julio de 2011). Diario Oficial No. 48.128 de 12 de julio de 2011. Bogotá, Colombia: Recuperado de: http://w ww.secretariasenado.gov.co/senado/bas edoc/ley_1474_2011.html.

Ley 1757. (06 de julio de 2015). Por la cual se dictan disposiciones en materia de promoción y protección del derecho a la participación democrática. Diario Oficial No. 49.565 dek Congreso de la República. Bogotá, Colombia.

Ley 489. (29 de diciembre de 1998). Diario Oficial No. 43. 464, de 30 de diciembre de 1998. Bogotá, Colombia: Recuperado de: http://www. secretariasenado.gov.co/senado/basedoc/ley_ 0489_1998.html.

Ley 850. (19 de noviembre de 2003). Por medio de la cual se reglamentan las veedurías ciudadanas. Diario Oficial No. 45.376 del Congreso de Colombia. Bogotá, Colombia: Recuperado de: http:// www.secretariasenado.gov.co/senado/basedoc/le y_0850_2003.html.

Machado, S. P., \& Montoya, O. D. (2021). Alcances, desarrollo y aportes de las veedurías ciudadanas en el control fiscal realizado por la Contraloría Municipal de Yumbo (Valle) durante la vigencia 2019-2020 (Trabajo de grado). Institución Universitaria Antonio José Camacho. Cali, Colombia. 
Suárez, C. L. (2017). Ejercicios de participación ciudadana: veeduría al Decreto 120 del 2007, frente al proceso de desarrollo urbano del municipio de Fusagasugá.https://repositorio.ucundinamarca.edu. co/bitstream/handle/20.500.12558/1582/Ejercicios \%20de\%20Participacion\%20Ciudadana\%20Veedu ria\%20\%20al\%20decreto\%20120\%20de\%202007\% 20\%20frente\%20al\%20desarrollo\%20urbano1.pdf? sequence $=1$ \&isAllowed $=\mathrm{y}$.

\section{AUTORES}

Julián Cocuy Espinosa: administrador de empresas, Especialista en Gerencia Estratégica y Magíster en Administración y Gestión Pública de la Universidad Santiago de Cali; Especialista en Desarrollo Empresarial de la Universidad Santo Tomás y Especialista en Gerencia de Proyectos de la Universidad del Tolima. Docente investigador de la Facultad de Ciencias Empresariales, Grupo de investigación GICES y Semillero Gestión E de la Institución Universitaria Antonio José Camacho de Cali-Valle. Correo electrónico: jcocuy@admon.uniajc.edu.co

Piedad Fernanda Machado Santacruz: abogada de la Universidad Santiago de Cali; Magíster en Políticas Públicas de la Universidad del Valle; Especialista en Gerencia de Instituciones Educativas de la Universidad del Tolima; Especialista en Administración Pública de la Universidad del Valle. Servidora pública, docente investigadora de la Facultad de Ciencias Empresariales, Grupo de Investigación GICES, Semillero Gestión E de la Institución Universitaria Antonio José Camacho de Cali-Valle. Correo electrónico: pmachado@admon.uniajc.edu.co

Daniela Montoya Ortiz: tecnóloga en Contabilidad Sistematizada de la Institución Universitaria Antonio José Camacho. Aspirante al título de Contadora Pública de la Institución Universitaria Antonio José Camacho. Integrante del Grupo de Investigación GICES y del Semillero Gestión E. Correo electrónico: dmontoyao@estudiantes.uniajc.edu.co 